Journal of Animal and Veterinary Advances 11 (13): 2250-2255, 2012

ISSN: $1680-5593$

(C) Medwell Journals, 2012

\title{
Seroepidemiological Survey of Equine Influenza a H3N8 in Horses from the Eastern Region of the United States-Mexico Border
}

\author{
${ }^{1}$ Ernesto J. Aguirre-Ezkauriatza, ${ }^{2}$ Erik A. Karlsson, ${ }^{2}$ Pamela Freiden, ${ }^{2}$ Stacey Schultz-Cherry, \\ ${ }^{3} \mathrm{~S}$. Gerardo H. Flores-Gutierrez and ${ }^{1}$ Manuel I. Zertuche-Guerra \\ ${ }^{1}$ Centro de Biotecnologia FEMSA, \\ Instituto Tecnologico y de Estudios Superiores de Monterrey, Monterrey, Nuevo Leon, Mexico \\ ${ }^{2}$ Department of Infectious Diseases, St. Jude Children's Research Hospital, \\ Memphis, Tennessee, United State \\ ${ }^{3}$ Facultad de Medicina Veterinaria y Zootecnia Dr. Norberto Trevino Zapata, \\ Universidad Autonoma de Tamaulipas, Mexico
}

\begin{abstract}
Seroprevalence studies of equine influenza virus in Mexico are very limited. For this reason, the main objective of this study is to start taking a series of samples looking for specific antibodies for equine influenza virus $\mathrm{AH} 3 \mathrm{~N} 8$ and the pandemic AH1N1 influenza virus. The higher seroprevalence of influenza A H3N8 Equine/Kentucky/CA09 was mainly associated with the dry season $(\mathrm{MH}-\mathrm{OR}=1.91 ; \mathrm{p}<0.0001)$, completely farmed horses $(\mathrm{MH}-\mathrm{OR}=1.554 ; \mathrm{p}<0.0001)$ with the permanent presence of dogs in the stables $(\mathrm{MH}-\mathrm{OR}=1.327$; $\mathrm{p}<0.0001)$, males and sgeldings $(\mathrm{MH}-\mathrm{OR}=1.239$ and 2.136 , respectively $\mathrm{p}<0.0001)$ of undefined breed and quarter horse cross breed $(\mathrm{MH}-\mathrm{OR}=1.367$ and 1.565 , respectively $\mathrm{p}<0.0001$ ) which were used for ridding and jumping $(\mathrm{MH}-\mathrm{OR}=1.274$ and 1.244 , respectively $\mathrm{p}<0.0001)$.
\end{abstract}

Key words: Equine influenza, AH3N8, AH1N1, seroprevalence, Eastern United States, Mexico border region

\section{INTRODUCTION}

The influenza A virus of the Orthomyxoviridae family affects a wide range of birds and mammals including human beings (Lorono-Pino et al., 2010). This etiological agent is classified into different subtypes based of its Haemagglutinin (HA) and Neuraminidase (NA) proteins, recognizing at the present day $16 \mathrm{HA}$ subtypes (H1-H16) and nine NA subtypes (N1-N9) (Daly et al., 2008). Equine Influenza (EI) is caused by $\mathrm{H} 7 \mathrm{H} 7$ or $\mathrm{H} 3 \mathrm{~N} 8$ subtypes. The H7N7 subtype was first identified in Czechoslovakia in 1956 (A/equine1/Prague/1/56) (Lorono-Pino et al., 2010) and at present time, it is considered as extinct among equines (Bogdan et al., 1993; Webster, 1993; Yamanaka et al., 2010).

The H3N8 subtype was first isolated from a horse in the USA in 1963 (A/equine2/Miami/1/63) and has been identified worldwide in horses (Wadell et al., 1963; Bogdan et al., 1993; Webster, 1993; Yamanaka et al., 2010) and is cause of several outbreaks (Daly et al., 2011). In addition, it has been recently demonstrated that horses could be a reservoir of H1N1 (Mancini et al., 2006). EI is cause of important economic losses due to its rapid spread among susceptible animals, high morbidity rate and its clinical signs which include depression, pyrexia, coughing, nasal discharge and neonatal losses also if EI is complicated by a secondary bacterial infection the affected animals can develop pneumonia and perish (El-Rahim and Hussein, 2004; Yamanaka et al., 2008; Lorono-Pino et al., 2010; Begg et al., 2011).

The equine population of Mexico comprises 11.57\% of the world census, however information concerning the presence and epidemiological patterns of EI is particularly scarce in this country. Consequently, the objective of this survey was to conduct a cross-sectionals in order to demonstrate the presence of EI and to identify some factors associated with the disease in the state of Tamaulipas, Mexico.

\section{MATERIALS AND METHODS}

Area description and biological samples: The state of Tamaulipas is located in Northeast Mexico, bordering the State of Texas in the United States of America. The survey was carried out the municipalities of Güemez and Victoria in the central area of Tamaulipas (Fig. 1).

Corresponding Author: Emesto J. Aguirre-Ezkauriatza, Centro de Biotecnologia FEMSA, Instituto Tecnologico y de Estudios Superiores de Monterrey, Ave. Eugenio Garza Sada 2501, Colonia Tecnologico, CP 64849, Monterrey, Nuevo Leon, Mexico 


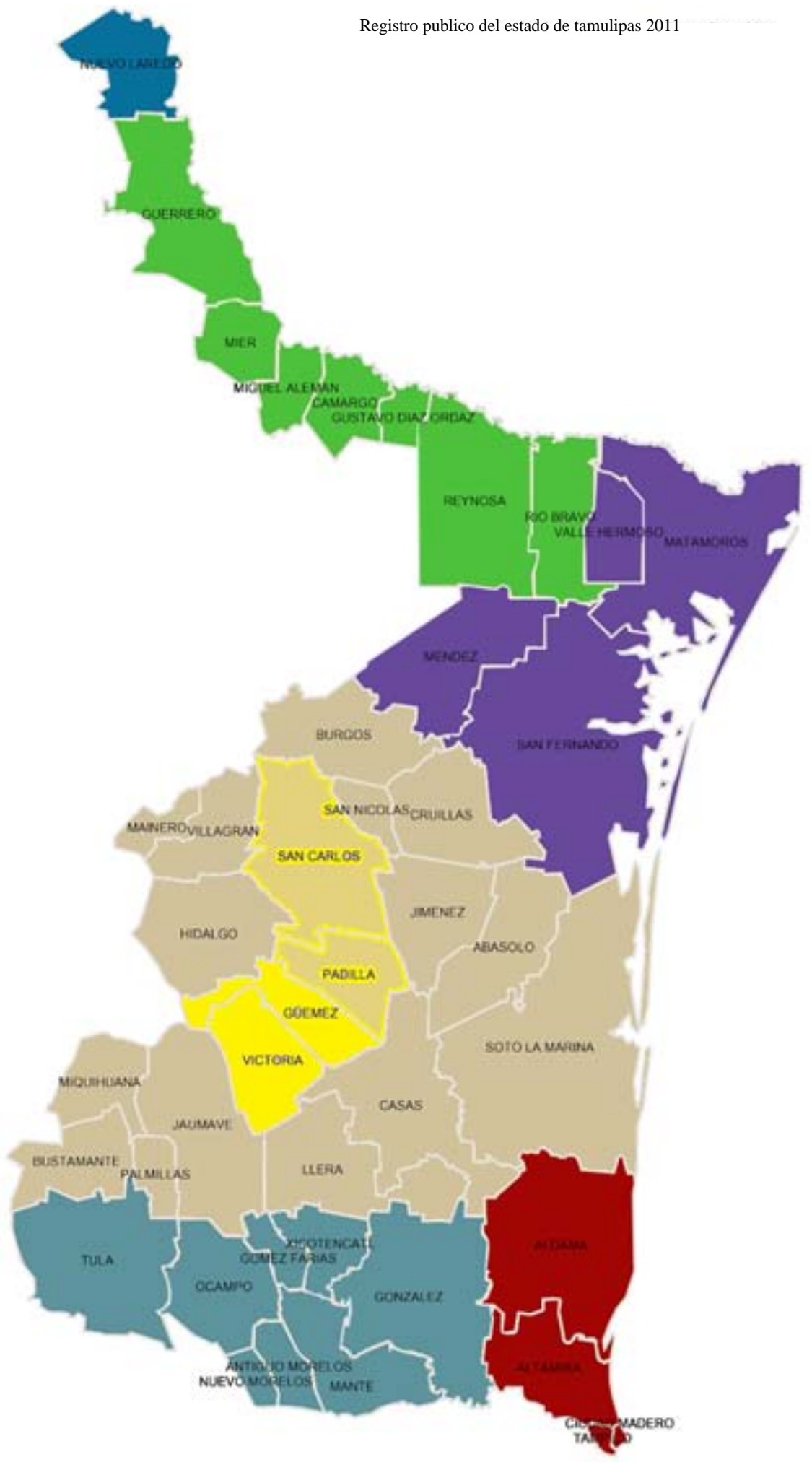

Fig. 1: Geographic location of: A) the state of Tamaulipas, Mexico; B) In solid yellow the municipalities included in the study area: a) Guemez; b) Victoria 
In agreement with the last animal population census provided by the Federal Government of Mexico, the average population of horses in both municipalities was of 262 heads which are mainly utilized for sporting and cattle-handling purposes.

Considering the facilities provided by the owners for carrying out the sampling process, 212 horses which never were vaccinated against equine influenza were included in the survey with this sample size, the probability of diagnosing at least one horse as positive was $98.74 \%$ as calculated with the software Win Episcope 2.0 (Acosta-Gonzalez et al., 2009). From April, 2010 to November, 2011, blood samples were collected by jugular venous puncture followed by clotting at room temperature for $4 \mathrm{~h}$ and stored at $4^{\circ} \mathrm{C}$ for $>3 \mathrm{~h}$ until their transport to laboratory.

Under laboratory conditions, the blood samples were centrifuged at $1,000 \times \mathrm{g}$ for $5 \mathrm{~min}$ at room temperature in order to obtain the serum samples required for carrying out immunological diagnostic tests. In addition, 80 nasal exudate samples were obtained using 17 inches swabs (Qosina, NY, USA) which were preserved in sterile M199 medium (Gibco ${ }^{\circledR}$, USA) $0.5 \%$ BSA supplemented with $2 \times 10^{6} \mathrm{U} \mathrm{L}^{-1}$ penicillin $\mathrm{G}, 200 \mathrm{mg} \mathrm{L}^{-1}$ streptomycin, $2 \times 10^{6} \mathrm{UL}^{-1}$ polymyxin $\mathrm{B}, 250 \mathrm{mg} \mathrm{L}^{-1}$ gentamicin, $0.5 \times 10^{6}$ $\mathrm{U} \mathrm{L}^{-1}$ nystatin, $60 \mathrm{mg} \mathrm{L}^{-1} \mathrm{HCL}$ ofloxacin and $0.2 \mathrm{~g} \mathrm{~L}^{-1}$ sulfamethoxazole. Once obtained, the nasal swabs also were stored at $4^{\circ} \mathrm{C}$ for $>3 \mathrm{~h}$ until transport to laboratory where were stored at $-80^{\circ} \mathrm{C}$ until their analysis. The total number of horses in the study was not sampled with nasal swabs because several owners considered this process a more invasive method than blood sampling and were not willing to allow for this kind of sampling.

Laboratory confirmation: The detection of non-specific antibodies against influenza $\mathrm{A}$ was realized at the Instituto Tecnologico y de Estudios Superiores de Monterrey in Monterrey, Mexico with an ELISA kit in agreement with the manufacturer's instructions (Virusys Corporation, Taneytown, MD, USA) each serum sample was analyzed by duplicate. Once non-specific antibodies against influenza $\mathrm{A}$ were detected, only the positive serum samples were further subjected to the hemagglutination inhibition assay at the Department of Infectious Diseases of the St. Jude Children's Research Hospital in Memphis, Tennessee, USA in order to detect specific antibodies against influenza A H3N8 equine/Kentucky/CA09 and influenza A H1N1 A/California/04/2009 as previously described. The use of ELISA as screening test followed by confirmation with Hemagglutination Inhibition assay $(\mathrm{HI})$ has demonstrated to have 93.98 and $98.71 \%$ of epidemiological sensitivity
Table 1: Prevalence and environmental risk factors associated with equine influenza in horses from Northeastern Mexico, near the United States-Mexico border region

\begin{tabular}{|c|c|c|c|c|}
\hline Variables & $\begin{array}{l}\text { Apparent } \\
\text { prevalence }\end{array}$ & $\begin{array}{c}\text { True } \\
\text { prevalence }\end{array}$ & $\begin{array}{c}\text { MH-OR* } \\
(\mathrm{p}<0.0001)\end{array}$ & $\mathrm{CI}(95 \%)$ \\
\hline \multicolumn{5}{|l|}{ Year of diagnosis } \\
\hline 2010 & 19.01 & 19.12 & 0.617 & \multirow[t]{2}{*}{$0.548-0.694$} \\
\hline 2011 & 25.32 & 25.93 & 1.621 & \\
\hline \multicolumn{5}{|l|}{ Season of year } \\
\hline Dry & 19.40 & 19.54 & 1.191 & \multirow[t]{3}{*}{$0.884-0.956$} \\
\hline Rainy & 17.65 & 17.65 & 0.817 & \\
\hline Cold & 19.30 & 19.43 & 0.919 & \\
\hline \multicolumn{5}{|l|}{ Municipality } \\
\hline Guemez & 22.45 & 22.83 & 1.270 & \multirow[t]{2}{*}{$0.76-0.816$} \\
\hline Victoria & 18.18 & 18.22 & 0.787 & \\
\hline \multicolumn{5}{|l|}{ Farming conditions } \\
\hline Stabled & 22.56 & 22.95 & 1.554 & \multirow[t]{6}{*}{$0.663-0.857$} \\
\hline Semi-stabled & 16.44 & 16.34 & 0.781 & \\
\hline Free grazing & 5.260 & 4.283 & 0.312 & \\
\hline Presence of dogs & 5.360 & 4.391 & 1.327 & \\
\hline Presence of poultry & 10.27 & 9.688 & 0.652 & \\
\hline \multicolumn{4}{|l|}{ Landscape } & \\
\hline Urban & 26.51 & 27.21 & 1.903 & \multirow[t]{3}{*}{$0.672-0.861$} \\
\hline Periurban & 16.00 & 15.87 & 0.761 & \\
\hline Rural & 13.43 & 13.10 & 0.596 & \\
\hline
\end{tabular}

*Mantel-Haenszel adjusted odds ratio

and specificity, respectively (De Benedictis et al., 2010). Also, at the St. Jude Children's Research Hospital, nasal swabs were subjected to the quantitative real time reverse-transcription PCR ( $\mathrm{PPCR}$ ). The positive control utilized in the qPCR was an EI commercial vaccine with the inactivated KY/97 strain (Fort Dodge ${ }^{\circledR}$, Iowa, USA).

Statistical analysis: The categorical variables of the diagnosed horses were the year of diagnosis, those variables related with the environment determinants under which the horses were exposed, i.e., season (dry, rainy and cold seasons), geographical origin (Güemez and Victoria municipalities), management conditions (stabled, semi-stables and free-grazing, permanent presence of dogs and domestic poultry in stables), landscape (urban, periurban and rural) and main use given to horses (racing, ridding, cattle handling, jumping and police horses) (Table 1). Also, there were variables which were statistically analyzed related to the host's determinant, i.e., gender (female, male and gelding), age (foal any gender, until 3 months), yearling (any gender from 4-24 months), filly (female, from 25-36 months), colt (male from 25-36 months), mare (adult female, 37 months and over), stallion (adult male, 37 and over) and gelding (orchidectomized male, 37 months and over)) and breed (undefined breed, Quarter horse, cross Quarter horse and cross thoroughbred) (Table 2).

In order to determine the apparent and true prevalence values of equine influenza, the population at risk considered was the total number of horses in each of the aforementioned variables. The association between diagnosis results and the aforementioned variables was 
Table 2: Prevalence and host-related risk factors associated with equine influenza in horses from Northeastern Mexico, near the United States, Mexico border region

\begin{tabular}{|c|c|c|c|c|}
\hline Variables & $\begin{array}{l}\text { Apparent } \\
\text { prevalence }\end{array}$ & $\begin{array}{c}\text { True } \\
\text { prevalence }\end{array}$ & $\begin{array}{c}\text { MH-OR* } \\
(p<0.0001)\end{array}$ & $\mathrm{CI}(95 \%)$ \\
\hline \multicolumn{5}{|l|}{ Gender } \\
\hline Female & 33.82 & 35.10 & 0.300 & $0.107-0.843$ \\
\hline Male & 45.24 & 47.42 & 1.239 & \\
\hline Gelding & 66.67 & 70.54 & 2.136 & \\
\hline \multicolumn{5}{|l|}{ Horse age } \\
\hline Yearling & 14.29 & 14.03 & 0.191 & $0.77-0.986$ \\
\hline Filly & 37.50 & 39.07 & 0.872 & \\
\hline Colt & 70.00 & 74.13 & 1.756 & \\
\hline Mare & 36.96 & 38.48 & 0.580 & \\
\hline Stallion & 62.50 & 66.04 & 1.542 & \\
\hline Gelding & 66.67 & 70.54 & 1.920 & \\
\hline \multicolumn{5}{|l|}{ Breed } \\
\hline Undefined & 50.00 & 52.55 & 1.367 & $0.478-0.629$ \\
\hline Quarter horse & 34.34 & 36.66 & 0.548 & \\
\hline Quarter horse cross & 58.02 & 61.20 & 1.565 & \\
\hline Thoroughbred cross & 42.86 & 44.85 & 0.815 & \\
\hline \multicolumn{5}{|l|}{ Use } \\
\hline Racing & 34.78 & 36.13 & 0.874 & $0.827-0.925$ \\
\hline Ridding & 47.96 & 50.35 & 1.357 & \\
\hline Cattle handling & 27.78 & 28.58 & 0.830 & \\
\hline Jumping & 50.00 & 52.55 & 1.325 & \\
\hline Police horse & 71.88 & 76.16 & 0.692 & \\
\hline
\end{tabular}

*Mantel-Haenszel adjusted odds ratio

determined using a stratified cross-sectional design this design was used in order to avoid the effect of confounding variables, i.e., horses' age, gender and geographical origin. The association was obtained with the Mantel-Haenszel statistical method for calculating the adjusted Odds Ratio (MH-OR). The analysis was carried out with the software Win Episcope 2.0 at a 95\% level of confidence, thus the Type I error was 0.05 .

\section{RESULTS AND DISCUSSION}

The main features of the sampled horses were the following: the age range was $12-240$ months (76.66 \pm 53.01 months) with $32.08,19.81$ and $39.62 \%$ of females, males and geldings, respectively. Concerning the farming conditions, the completely stabled individuals predominated $(62.74 \%$ ) followed by semi-stabled horses $(34.43 \%)$ and animals that were under free grazing conditions $(8.96 \%)$. The quarter horse breed predominated $(46.7 \%)$ and was followed by quarter horse cross breed $(38.21 \%)$, undefined breed (17.92\%) and thoroughbred cross breed $(3.3 \%)$. The main use of these horses was for riding $(46.23 \%)$, racing $(32.55 \%)$ and police horses $(15.09 \%)$; a lesser percent of the horses were used for cattle handling $(8.49 \%)$ and jumping $(3.77 \%)$.

The overall seroprevalence of non-specific antibodies against influenza A H3N8 was $45.78 \%$. When the positive samples were analyzed, the seroprevalence of specific antibodies against influenza A H3N8 Equine/ Kentucky/CA09 was $19.11 \%$ which was higher in the year
$2011(25.32 \%)$ for the municipality of Guemez $(22.45 \%)$ and for geldings $(26.19 \%)$, however the seroprevalence values slightly varied when was recalculated using the diagnostic confidence limits reported by De Benedictis et al. (2010) (Table 1). The higher seroprevalence of influenza A H3N8 Equine/Kentucky/CA09 antibodies was mainly associated with the dry season $(\mathrm{MH}-\mathrm{OR}=1.91 ; \mathrm{p}<0.0001)$ completely stabled horses $(\mathrm{MH}-\mathrm{OR}=1.554 ; \mathrm{p}<0.0001)$ under urban landscape $(\mathrm{MH}-\mathrm{OR}=1.903 ; \mathrm{p}<0.0001)$ and with the permanent presence of dogs in the stables $(\mathrm{MH}-\mathrm{OR}=1.327 ; \mathrm{p}<0.0001)$.

The presence of seropositive horses also was associated with males and geldings $(\mathrm{MH}-\mathrm{OR}=1.239$ and 2.136 , respectively $\mathrm{p}<0.0001$ ) of undefined breed and quarter horse cross breed $(\mathrm{MH}-\mathrm{OR}=1.367$ and 1.565 , respectively; $\mathrm{p}<0.0001$ ) and those animals which were used for ridding and jumping $(\mathrm{MH}-\mathrm{OR}=1.274$ and 1.244, respectively; $\mathrm{p}<0.0001$ ). No positive results were obtained when serum samples were subjected to $\mathrm{HI}$ to detect antibodies against the reference strain H1N1 A/California/04/2009 nor when the nasal swabs were subjected to $\mathrm{qPCR}$ in order to detect Influenza A non specific strain. EI is considered a highly pathogenic disease for equines and it results in severe economic losses mainly due to cancelation of sporting and racing events which has been demonstrated in several outbreaks around the world (Yamanaka et al., 2008). In this century, EI outbreaks have occurred in Croatia, Japan (Nishiura and Satou, 2010) India, Mongolia, China (Virmani et al., 2010). In agreement with the World Organization of Animal Health (OIE), the last EI outbreak in Mexico was recorded in 1997 and after this year, the presence of the disease is suspected but not confirmed by the animal health authorities.

Some epidemiological patterns of EI have been determined during outbreaks, however because the aforementioned analysis have been done using a purposive sampling that involves diseased animals, the generated information could not be representative of the general population and the findings only can be extrapolated to equines with clinical signs. In spite of this, the seroprevalence determined in this study was $<22.85 \%$ reported for outbreaks in India (Virmani et al., 2010), 37\% in Hong Kong (Lai et al., 1994) $56.5 \%$ in Canada (Diaz-Mendez et al., 2010) and 5.6\% in Egypt (El-Rahim and Hussein, 2004) however, the results of this study can be compared and were similar to the $19.4 \%$ reported by Yamanaka et al. (2008) whom analyzed subclinical healthy race horses in an outbreak in Japan but when compared with the results of race horses in this study were considerable higher than the aforementioned 
study. On the other hand, the seroprevalence rate of this study was $>13.6 \%$ in Tunisia (Boussetta et al., 1994) and 9.07\% in the United States of America (Pusterla et al., 2011) reported in random surveillance surveys. In Mexico, previous surveys have reported seroprevalence rates of $28 \%$ (Lorono-Pino et al., 2010) and 38.8\% (Blitvich et al., 2010) both seroprevalence values are higher than the reported in this study. In addition in the aforementioned reports, the seroprevalence was not associated with risk factors.

Concerning risk factors there is a great lacking of information, probably due to the urgency for controlling outbreaks rather than generating epidemiological information. In spite of this, the presence of EI has been associated with the animals' age. In the United Kingdom, Newton et al. (2006) reported that infections among 2 years old horses were lower than in older animals which is in agreement with the risk factors determined in this study. In this study, older animals were also associated with the presence of antibodies against H3N8 equine/Kentucky/CA09 which is in disagreement with the report of Goto et al. (1993) whom reported that in Japan the presence of reactors decreased in older horses.

Racing horses also has been previously associated with EI (Lany et al., 1997), however in this study, the aforementioned horses' activity was not determined as a risk factor but ridding and jumping horses were strongly associated with the disease because under local conditions hundreds and thousands in some particular circumstances, of horses are in close contact in these activities. Another factor associated with EI has been the stabled horses (Powell et al., 1995) which is in agreement with the results of this study horses that were located in periurban locations also are associated with high seroprevalence rates (Cowled et al., 2009) similarly to the horses located in urban locations in this study. Also, Yamanaka et al. (2008) in Japan and Blitvich et al. (2010) and Lorono-Pino et al. (2010) in Mexico, determined differences in morbidity rates in geographical location as in this study.

Recent evidence exists showing that dogs can be infected by different EI virus strains when they are in close contact with infected horses (Daly et al., 2008; Hayward et al., 2010; Kirkland et al., 2010; Crispe et al., 2011). Despite the fact that the role of dogs has not been clearly demonstrated in the epidemiology or perpetuation of the disease this study reports an association between EI in horses that were permanently in close contact with dogs. Also, wild birds are considered a natural reservoir of EI virus (Daly et al., 2008) and these could transmit the disease to horses (Spokes et al., 2009) and domestic poultry, however the permanent presence of domestic poultry in the analyzed stables was not associated with the reported seroprevalence rates. Despite the use of effective control measures, i.e., vaccinations programs, movement restriction, isolation and quarantine of infected horses or stables (Nishiura and Satou, 2010) when outbreaks take place, EI due to the influenza is still a significant health problem worldwide. Continuous surveillance is necessary in order to detect clinical or subclinical cases or antigenic drift of the virus. Thus, this study emphasizes the need to conduct epidemiological surveys in randomly selected populations of equines in order to determine not only risk factors but also to implement early warning systems for the prevention and control of outbreaks as well as implementing the appropriate eradication programs.

\section{CONCLUSION}

No positive results were obtained when serum samples were subjected to $\mathrm{HI}$ to detect antibodies against the reference strain A H1N1 A/California/04/2009.

\section{ACKNOWLEDGEMENTS}

This study is part of the doctoral thesis of Ernesto J. Aguirre-Ezkauriatza who was a recipient of a Doctoral Scholarship provided by the Consejo Nacional de Ciencia y Tecnologia (Mexico). The study was supported by the St. Jude Children's Research Hospital and Centro de Biotecnologia FEMSA of the Instituto Tecnologico y de Estudios Superiores de Monterrey (Mexico). The researchers thank Dr. Emiliano Salatino for his critical reading of the manuscript.

\section{REFERENCES}

Acosta-Gonzalez, R.I., F. Infante and G.H. FloresGutierrez, 2009. Epidemiological patterns of caprine brucellosis in an unvaccinated area, Mexico. Rev. Med. Vet., 3: 145-148.

Begg, A.P., R.L. Reece, S. Hum, W. Townsend, A. Gordon and J. Carrick, 2011. Pathological changes in horses dying with equine influenza in Australia, 2007. Aust. Vet. J., 89: 19-22.

Blitvich, B.J., L.A. Ibarra-Juarez, A.J. Cortes-Guzman, J.J. Root, A.B. Franklin, H.J. Sullivan and I. Fernandez-Salas, 2010. Seroprevalence of equine influenza virus in North-East and Southern Mexico. Vet. Rec., 166: 565-566.

Bogdan, J.R., P.S. Morley, H.G. Townsend and D.M. Haines, 1993. Effect of influenza A/equine/H3N8 virus isolate variation on the measurement of equine antibody responses. Can. J. Vet. Res., 57: 126-130. 
Boussetta, M., A. Chabchoub, A. Ghram, I. Jomaa, A. Ghorbel, T. Aouina and H.B. Amor, 1994. Seroepidemiological survey of influenza and infectious anemia in Equidae in northeastern Tunisia. Rev. Elev. Med. Vet. Pays. Trop., 47: 277-281.

Cowled, B., M.P. Ward, S. Hamilton and G. Garner, 2009. The equine influenza epidemic in Australia: Spatial and temporal descriptive analyses of a large propagating epidemic. Prev. Vet. Med., 92: 60-70.

Crispe, E., D.S. Finlaison, A.C. Hurt and P.D. Kirkland, 2011. Infection of dogs with equine influenza virus: Evidence for transmission from horses during the Australian outbreak. Aust. Vet. J., 89: 27-28.

Daly, J.M., A.S. Blunden, S. Macrae, J. Miller and S.J. Bowman et al., 2008. Transmission of equine influenza virus to English foxhounds. Emerg. Infect. Dis., 14: 461-464.

Daly, J.M., S. MacRae, J.R. Newton, E. Wattrang and D.M. Elton, 2011. Equine influenza: A review of an unpredictable virus. Vet. J. Lond. England, 189: 7-14.

De Benedictis, P., T.C. Anderson, A. Perez, E. Viale and C. Veggiato et al., 2010. A diagnostic algorithm for detection of antibodies to influenza: A viruses in dogs in Italy (2006-2008). J. Vet. Diagn. Invest., 22: 914-920.

Diaz-Mendez, A., L. Viel, J. Hewson, P. Doig and S. Carman et al., 2010. Surveillance of equine respiratory viruses in Ontario. Can. J. Vet. Res., 74: 271-278.

El-Rahim, A.I.H. and M. Hussein, 2004. An epizootic of equine influenza in Upper Egypt in 2000. Rev. Sci. Tech., 23: 921-930.

Goto, H., Y. Yamamoto, C. Ohta, T. Shirahata, T. Higuchi and H. Ohishi, 1993. Antibody responses of Japanese horses to influenza viruses in the past few years. J. Vet. Med. Sci., 55: 33-37.

Hayward, J.J., E.J. Dubovi, J.M. Scarlett, S. Janeczko, E.C. Holme and C.R. Parrish, 2010. Microevolution of canine influenza virus in shelters and its molecular epidemiology in the United States. J. Virol., 84: 12636-12636.

Kirkland, P.D., D.S. Finlaison, E. Crispe and A.C. Hurt, 2010. Influenza virus transmission from horses to dogs, Australia. Emerg. Infect. Dis., 16: 699-702.
Lorono-Pino, M.A., J.A. Farfan-Ale, J.E. Garcia-Rejon, M. Lin and E. Rosado-Paredes et al., 2010. Antibodies to influenza and West Nile viruses in horses in Mexico. Vet. Rec., 166: 22-23.

Mancini, D.A.P., E.M.S. Cunha, M.C.C.S.H. Lara, R.M.Z. Mendonca and A.L.F. Dias et al., 2006. Evidence of human-equine interspecies influenza virus transmission. Virus Rev. Res., 11: 44-50.

Newton, J.R., J.M. Daly, L. Spencer and J.A. Mumford, 2006. Description of the outbreak of equine influenza (H3N8) in the United Kingdom in 2003, during which recently vaccinated horses in Newmarket developed respiratory disease. Vet. Rec., 158: 185-192.

Nishiura, H. and K. Satou, 2010. Potential effectiveness of public health interventions during the equine influenza outbreak in racehorse facilities in Japan, 2007. Transbound. Emerg. Dis., 57: 162-170.

Powell, D.G., K.L. Watkins, P.H. Li and K.F. Shortridge, 1995. Outbreak of equine influenza among horses in Hong Kong during 1992. Vet. Rec., 136: 531-536.

Pusterla, N., P.H. Kass, S. Mapes, C. Johnson and D.C. Barnett et al., 2011. Surveillance programme for important equine infectious respiratory pathogens in the USA. Vet. Rec., 169: 12-12.

Spokes, P.J., A.J. Marich, J.A. Musto, K.A. Ward, A.T. Craig and J.M. McAnulty, 2009. Investigation of equine influenza transmission in NSW: Walk, wind or wing?. N. S. W. Public Health Bull., 20: 152-156.

Virmani, N., B.C. Bera, B.K. Singh, K. Shanmugasundaram and B.R. Gulati et al., 2010. Equine influenza outbreak in India (2008-09): Virus isolation, sero-epidemiology and phylogenetic analysis of $\mathrm{HA}$ gene. Vet. Microbiol., 143: 224-237.

Wadell, G.H., M.B. Teigland and M.M. Sigel, 1963. A new influenza virus associated with equine respiratory disease. J. Am. Vet. Med. Assoc., 143: 587-590.

Webster, R.G., 1993. Are equine influenza viruses still present in horses?. Equine Vet. J., 25: 537-538.

Yamanaka, T., H. Niwa, K. Tsujimura, T. Kondo and T. Matsumura, 2008. Epidemic of equine influenza among vaccinated racehorses in Japan in 2007 . J. Vet. Med. Sci., 70: 623-625.

Yamanaka, T., K. Tsujimura, T. Kondo, T. Matsumura and H. Ishida et al., 2010. Infectivity and pathogenicity of canine H3N8 influenza A virus in horses. Influenza Respir. Viruses, 4: 345-351. 